Psychotherapeut 2022 $67: 158-165$

https://doi.org/10.1007/s00278-022-00576-z

Angenommen: 23. Dezember 2021

Online publiziert: 7. Februar 2022

() Der/die Autor(en) 2022

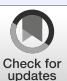

\section{Klang der Depression}

\section{Zusammenhang zwischen Depressivität und paraverbalen Merkmalen während der Anamnese}

Alisa Shugaley · Uwe Altmann · Maria Brümmel · Julija Meier · Bernhard Strauß . Désirée Schönherr

Institut für Psychosoziale Medizin, Psychotherapie und Psychoonkologie, Universitätsklinikum Jena, Jena, Deutschland

\section{Zusammenfassung}

\section{In diesem Beitrag}

\author{
- Grundlagen \\ Paraverbale Merkmale in medizinischem \\ und psychotherapeutischem Kontext • \\ Paraverbale Korrelate der Depression \\ - Ziel der Arbeit \\ - Material und Methoden \\ Datenerhebung und Design - Stich- \\ probe und Ablauf - Erhobene Maße • \\ Datenanalyse \\ - Ergebnisse \\ - Diskussion \\ Interpretation der Ergebnisse - Stärken \\ und Limitationen der Studie
}

\section{Zusatzmaterial online}

Die Online-Version dieses Beitrags (https:// doi.org/10.1007/s00278-022-00576-z) enthält 2 weitere Tabellen sowie eine zusätzliche Abbildung.

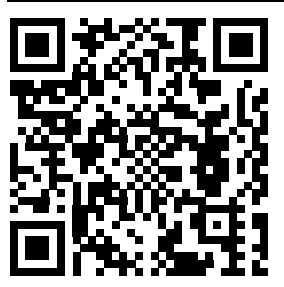

QR-Code scannen \& Beitrag online lesen

Hintergrund: Die Depression ist neben kognitiven, emotionalen, sozialen und psychomotorischen Beeinträchtigungen durch spezifische stimmliche Merkmale gekennzeichnet. Bisher existieren erst wenige Studien, die diese in klinischen Kontexten und in Abgrenzung zu Gesunden untersuchten.

Fragestellung: Untersucht wurde der Zusammenhang zwischen Depressivität und paraverbalen Merkmalen bei depressiven Patient_innen und gesunden Probanden. Methode: In einem mehrschrittigen Verfahren wurden die Audioinhalte von Anamneseinterviews mit Depressiven $(n=15)$ und Gesunden $(n=15)$ mit einer Software annotiert und transkribiert. Die paraverbalen Merkmale Grundfrequenz der Stimme, Spannweite der Stimme, Sprechgeschwindigkeit und Pausenlänge wurden automatisiert bestimmt. Mithilfe hierarchisch linearer Modelle wurde der Einfluss der Gruppenzugehörigkeit, Depressivität, Ängstlichkeit sowie psychischer und körperlicher Gesundheit auf die paraverbalen Merkmale analysiert.

Ergebnisse: Es zeigte sich ein Zusammenhang zwischen Depressivität und der Sprechgeschwindigkeit. Zwischen der Spannweite der Grundfrequenz, der Pausenlänge und Depressivität konnten tendenzielle Zusammenhänge gefunden werden. Depressive Patient_innen sind im Vergleich zu Gesunden durch eine monotone Sprache, eine geringe Sprechgeschwindigkeit und längere Pausen gekennzeichnet. Sprechgeschwindigkeit und Pausenlänge waren ebenfalls mit Ängstlichkeit assoziiert. Diskussion: Sprechgeschwindigkeit, Pausenlänge und Spannweite der Grundfrequenz scheinen relevante Indikatoren für Depressivität/ggf. Ängstlichkeit zu sein.

Die Spannweite der Grundfrequenz ist eher depressionsspezifisch, während Pausenlänge und Sprechgeschwindigkeit mit Depressivität und Ängstlichkeit assoziiert sind. Zukünftige Studien sollten die Zusammenhänge in größeren Stichproben verschiedener klinischer Störungsbilder untersuchen.

\section{Schlüsselwörter}

Sprachproduktionbewertung · Stimmanalyse - Verbales Verhalten · Nonverbale Kommunikation . Ängstlichkeit
Die Diagnose Depression wird aufgrund von Bewertungen verschiedener Ebenen gestellt (kognitiv, emotional, verhaltensbezogen). Die nonverbale und die paraverbale Ebene werden bei diagnostischen Entscheidungen implizit bewertet, aber in Diagnosemanualen nicht differenziert. Studien aus jüngerer Zeit zeigen eindrücklich die Bedeutung nonverbalen und paraverbalen Verhaltens im Kontext psychischer Erkrankungen (Wiltshire et al. 2020), wobei die Berück- sichtigung speziell paraverbaler Merkmale für diagnostische Entscheidungen noch genauer betrachtet werden sollte (Juslin und Scherer 2005).

\section{Hintergrund}

Untersuchungen zur Bedeutung paraverbaler Merkmale für diagnostische Entscheidungen könnten eine Überführung der aktuell implizit genutzten Merkmale in Diagnosemanuale ermöglichen. Defi- 
nitionen expliziter paraverbaler Kriterien können zu besserer Übereinstimmung und Kommunikation zwischen Diagnostikern, zur Entwicklung standardisierter Erhebungsinstrumente und zu einer höheren Qualität der Ausbildung führen, da diese auf explizite Maße im Gegensatz zu subjektiven Empfindungen zurückgreifen können (Jablensky und Kendell 2002).

\section{Grundlagen}

Paraverbale Merkmale in medizinischem und psychotherapeutischem Kontext

Eine gelungene Kommunikation kann die Beziehung zwischen den Interaktionspartnern sowie den Behandlungserfolg in ärztlichen (Roter et al. 2006) und psychotherapeutischen Gesprächen (Westland 2015) positiv beeinflussen (Faller 2010; Lindemann 2015). Kommunikation findet auf verschiedenen Ebenen statt: linguistisch/ verbal (sprachliche Inhalte), extralinguistisch (Mimik, Gestik, Körperkinetik usw.) oder paralinguistisch/paraverbal (Lautstärke, Stimmlage etc.; Tonti und Gelo 2016). Dabei werden die extralinguistischen und die paraverbalen Merkmale dem Oberbegriff des nonverbalen Verhaltens zugeordnet (Argyle 1979). Bis heute überwiegen Untersuchungen zur verbalen und speziell zur extralinguistischen Kommunikation (Schuller et al. 2013).

Paraverbale Phänomene spielen nicht nur beim Ausdruck und bei der Wahrnehmung von Emotionen eine zentrale Rolle, sondern geben auch Aufschluss über Aspekte wie Gesundheitszustand, Stresserleben oder Motivation der sprechenden Person (Schuller et al. 2013). Emotionsbezogene Kommunikationsfertigkeiten, wie das Wahrnehmen paraverbaler Informationen und das Bewusstsein für das Senden solcher Signale, beeinflussen die Qualität der medizinischen Betreuung erheblich (Pennacchini und Pensieri 2011; Roter et al. 2006). Dabei ist auffällig, dass $95 \%$ der Studien zu nonverbalen Merkmalen fast ausschließlich die Mimik im Kontext von psychischen Störungen untersuchen und paraverbalen Merkmalen nur geringe Aufmerksamkeit zukommt (Balsters et al. 2012). Diese Tatsache geht nicht konform mit der Empfehlung, Interaktionen auf al- len Kommunikationsebenen zu betrachten, um die Informationsgewinnung zu maximieren und trennschärfere Diagnosen zu stellen (Pennacchini und Pensieri 2011).

\section{Paraverbale Korrelate der Depression}

Paraverbale Merkmale können zusätzliche Indikatoren zur Beurteilung bestehender Depression sein (Cummins et al. 2015). Mehrheitlich wird ein Zusammenhang zwischen reduzierter $F_{0}$-Spannweite $\left(F_{0}\right.$ : Grundfrequenz der Stimme) und erhöhter Depressivität beschrieben (Cannizzaro et al. 2004; Mundt et al. 2007). Auch wird mehrfach eine Korrelation zwischen reduzierter Sprechgeschwindigkeit und dem Schweregrad der Depression berichtet (Cannizzaro et al. 2004; Mundt et al. 2012). Einerseits werden Zusammenhänge zwischen Behandlungserfolg und Steigerung der $F_{0}$ bei Patient_innen mit Depression berichtet (Mundt et al. 2012), andererseits wurden keine signifikanten Gruppenunterschiede zwischen Patient_innen mit Depression und Gesunden bezüglich der $F_{0}$ beschrieben (Taguchi et al. 2018). Dabei ist anzumerken, dass sich das Stimulusmaterial der beiden Studien deutlich unterschied: Mundt et al. (2012) untersuchten Gesprächsproben aus Telefonaten, Taguchi et al. (2018) das kontrollierte Vorlesen von Ziffern unter Laborbedingungen. Eine längere Pausendauer (i.e. Pausen zwischen Äußerungen) kann als der bisher vielversprechendste Indikator für die Depression angesehen werden, da dies über mehrere Studien übereinstimmend gezeigt werden konnte (Balsters et al. 2012).
Hier steht eine Anzeige.

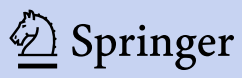




\section{Schwerpunkt: Sprache als Medium in Psychotherapien - Originalien}

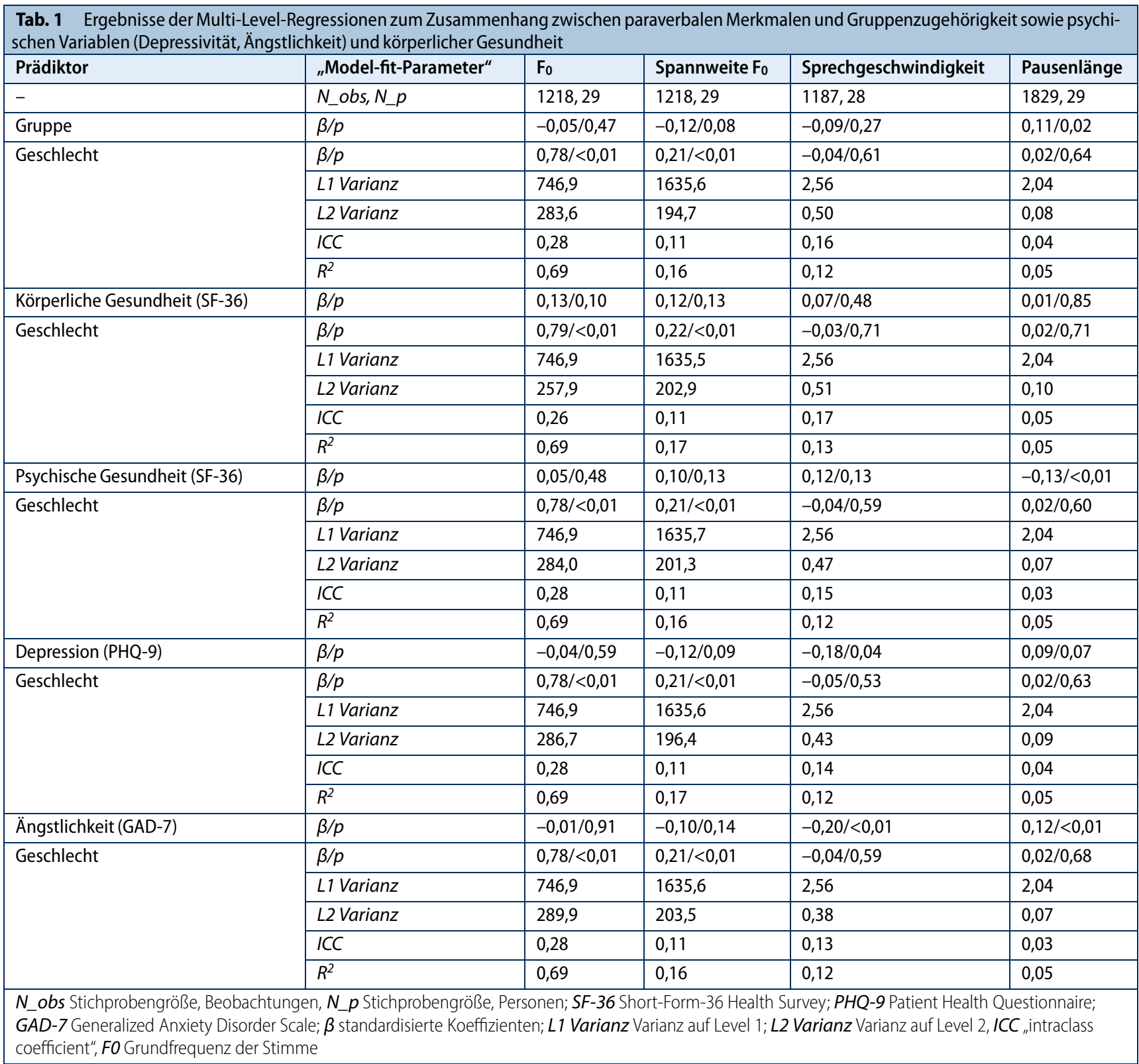

\section{Ziel der Arbeit}

Im primären Fokus der vorliegenden Untersuchung stand der Vergleich einer psychiatrischen Stichprobe depressiver Patient_innen mit einer gesunden Kontrollgruppe im Hinblick auf paraverbale Merkmale. Als paraverbale Merkmale wurden die $F_{0}$, ihre Spannweite, die Sprechgeschwindigkeit und Pausenlänge untersucht. Bisherige Studien zum Zusammenhang zwischen Depressivität und paraverbalen Merkmalen untersuchten dies stets unter Laborbedingungen, mit standardisiertem Material oder in Mo- nologen von Patienten, sodass ein Transfer in den natürlichen Kontext erschwert ist.

\section{Material und Methoden}

\section{Datenerhebung und Design}

Die Untersuchung wurde als Sekundäranalyse einer Pilotstudie durchgeführt, die im quasiexperimentellen Design ohne Messwiederholung die Bedeutung der Synchronie von Körperbewegungen und Mimik in der Patient-Arzt-Kommunikation bei depressiven und gesunden Probanden untersuchte (Altmann et al. 2020a; 2020b). Die aktuelle Untersuchung ergänzt die vor- handenen Ergebnisse um Analysen paraverbaler Stimmparameter.

\section{Stichprobe und Ablauf}

An der Primärstudie nahmen insgesamt 30 Personen (20 bis 30 Jahre alt) teil, davon 15 Gesunde und 15 Patient_innen in stationärer Behandlung mit einer zum Studienzeitpunkt diagnostizierten depressiven Episode (unipolar nach Kriterien des ICD-10). Die Rekrutierung wurde in 2 Stufen durchgeführt: Erst wurde ein_e Patient_in mithilfe des Chef- und Oberarztes der Station für affektive Störungen des Universitätsklinikums Jena 
akquiriert. Einschlusskriterien waren das Vorliegen einer unipolaren depressiven Episode sowie ein Alter zwischen 20 und 30 Jahren. Es gab keine Restriktionen hinsichtlich der Komorbiditäten. Im zweiten Schritt wurde eine Kontrollperson durch Aushänge und Nutzen der sozialen Medien gesucht, die der/dem Patient_in in Geschlecht und Alter ( \pm 3 Jahre) glich. Somit wurde die Vergleichbarkeit beider Gruppen nach Geschlecht und Alter gewährleistet (genaue Informationen zu Rekrutierung und Studienfluss: Altmann et al. 2020a). Das Alter der Stichprobe betrug im Mittel 25,2 Jahre (Standardabweichung $[S D] \pm 3,1$ Jahre; $M_{K G}=25,5$ Jahre, $S D_{K G} \pm 3,3$ Jahre; $M_{\text {Pat }}=24,9$ Jahre, $\mathrm{SD}_{\text {Pat }} \pm 3,1$ Jahre). In beiden Gruppen befanden sich $60 \%$ Männer und $40 \%$ Frauen. Mindestens das Abitur hatten $80 \%$ der Stichprobe $\left(\mathrm{n}_{\mathrm{KG}}=93,3 \% ; \mathrm{n}_{\text {Pat }}=66,7 \%, X^{2}\right.$ $(1)=3,3 ; V=0,333, p<0,05)$. In einer Partnerschaft befanden sich aktuell $26,7 \%$ $\left(n_{K G}=33,3 \% ; n_{\text {Pat }}=20,0 \%\right)$. Ausschlusskriterien für die klinische Stichprobe waren starke Einschränkungen der Kommunikation (Störungen der Wahrnehmung, des Seh- oder Hörvermögens nach subjektiver Einschätzung sowie mangelnde Deutschkenntnisse). Personen mit eingeschränkter Beweglichkeit der KopfNacken-Region oder der Mimik, einer bestehenden manischen Episode, psychotischen Symptomen oder einer zu hohen Eigengefährdung wurden ebenfalls ausgeschlossen. Ausschlusskriterien für die gesunde Stichprobe waren: Medikation mit Psychopharmaka, diagnostizierte psychische Erkrankungen, Inanspruchnahme einer psychiatrischen oder psychotherapeutischen Behandlung oder starke Kommunikationseinschränkungen (Altmann et al. 2020a, b). Technische Einschlusskriterien waren: existierende Audiospuren und eine gute Qualität des Audiomaterials (wenig Hintergrundrauschen, kein starker Dialekt). Es mussten keine Dyaden aufgrund technischer Kriterien ausgeschlossen werden.

Alle aufgezeichneten Anamnesegespräche unterlagen den gleichen Aufnahmebedingungen (selber Raum, gleiche Sitz- und Kamerapositionierung, Kamera der Marke Canon Legria HFM52, Canon, Tokio, Japan) und dem gleichen Ablauf (Begrüßung, Fragebogen ausfüllen, Sek- tion G "Somatoforme Störungen“ aus dem Strukturierten Klinischen Interview für die Diagnose psychischer Störungen der Achse I des DSM-IV [SKID-I]). Sektion $\mathrm{G}$ wurde verwendet, da auch gesunde Probanden gelegentlich über körperliche Beschwerden klagen. Dementsprechend wurde in diesem Abschnitt eine Annäherung der Gesprächsanteile der beiden Gruppen erwartetet. Die Videos der Anamnesegespräche wurden auf die Fragen G16-G31 (Schmerzsymptome und gastrointestinale Symptome - Details: Zusatzmaterial online: Tabelle S1) des SKID-I zugeschnitten, weil selbst gesunde Menschen gelegentlich über Schmerzen oder Verdauungsbeschwerden klagen (Häuser et al. 2013). Es handelt sich nur um einen Teil des Interviews, das für die Analysen der Bewegungs- und Mimikparameter genutzt wurde (Altmann et al. 2020a, b). Die Audiospuren wurden mithilfe eines VLC Players (Version 2.2.6) extrahiert und formatiert (Abtastrate $44.000 \mathrm{~Hz}, 2$ Kanäle, Bitrate 128). Die konvertierten Audiodateien wurden in das Sprachanalyseprogramm Praat (Version 6.0.46; Boersma 2001) geladen und von einer kodierenden Person weiterverarbeitet. In einer TextGrid-Datei wurden die manuelle Segmentierung der Personensprechanteile (Annotation) und der Sprechpausen sowie die Transkription der gesprochenen Inhalte auf Grundlage von Kodierregeln (Zusatzmaterial online: Tabelle S2) gespeichert.

Die Studie wurde von der lokalen Ethikkommission bewilligt (ID 5043-01/17). Von allen beteiligten Patient_innen liegt eine Einverständniserklärung vor. Die Freiwilligkeit der Studienteilnahme wurde stets betont.

\section{Erhobene Maße}

\section{Fragebogen}

Es wurde die gesundheitsbezogene Lebensqualität mit dem Short-Form-36 Health Survey (SF-36; Bullinger et al. 1995), die Depressivität mit dem Patient Health Questionnaire (PHQ-9; Kroenke et al. 2001) und die Angstsymptomatik mithilfe der Generalized Anxiety Disorder Scale (GAD-7; Spitzer et al. 2006) erhoben. Für die Sekundäranalyse war v.a. der PHQ-9 von besonderem Interesse. Aufgrund der hohen Komorbidität von Depression mit Ängstlichkeit sowie Somatisierungen wurden die oben genannten Fragebogen zusätzlich einbezogen. Anzumerken ist, dass Studien, die die Ängstlichkeit im Zusammenhang mit paraverbalen Merkmalen untersuchten, eine höhere $F_{0}$ berichten; diese ist mit einem höheren Arousal assoziiert (Weeks et al. 2012).

Der PHQ-9 besteht aus 9 Items und misst die Häufigkeit depressiver Symptome nach den Kriterien des DSM-IV für die Major Depression während der vergangenen 2 Wochen. Die Beantwortung der Items erfolgt auf einer 4-stufigen Skala. Die Summenwerte von 0 bis 27 können als Kontinuum gesehen werden, bei dem Werte von 5-9 eine milde, von 10-14 eine moderate, von 15-19 eine mittelgradige und von 20-27 eine schwere depressive Symptomatik repräsentieren.

Mithilfe des SF-36 werden die selbsteingeschätzte gesundheitsbezogene Lebensqualität und die subjektive Gesundheit erfasst. Acht verschiedene Skalen der körperlichen und psychischen Gesundheit werden durch 35 Items in mehreren Antwortformaten erfragt. Das 36. Item erfragt die Veränderung des Gesundheitszustandes zum Vorjahr (genaue Informationen: Bullinger et al. 1995). In der vorliegenden Studie wurden die Summenskalen psychische Gesundheit sowie körperliche Gesundheit des SF-36 genutzt.

Die GAD-7 erfasst mithilfe von 7 Items mit 4-stufigem Likert-Skalen-Niveau die Ängstlichkeit der Patient_innen in Anlehnung an die Kriterien der generalisierten Angststörung des DSM-IV (Spitzer et al. 2006).

\section{Paraverbale Merkmale}

Um den Einfluss möglicher Ausreißer zu reduzieren, wurde für die $\mathrm{F}_{0}$ der Median analysiert. Die $\mathrm{F}_{0}$ wurde in $\operatorname{Hertz}(\mathrm{Hz})$ gemessen. Die Spannweite der $F_{0}$ repräsentiert das Ausmaß der Monotonie im Stimmklang. Um die Verzerrung durch Hintergrundgeräusche zu reduzieren, wurde die Spannweite der $\mathrm{F}_{0}$ als Differenz des 5\%- und 95\%-Quantils der $F_{0}$ operationalisiert (Schoenherr et al. 2021). Da die Spontansprache viele Unterbrechungen unter 200 ms beinhaltet (Stassen 1995), wurden nur Sprechpausen, deren Länge den Schwellenwert von 1s überschritt, be- 
rücksichtigt. Die Sprechgeschwindigkeit wurde als Quotient aus Silbenzahl und Dauer des Sprechanteils (Sprechanteil: Silben/s) operationalisiert. Die paraverbalen Parameter (Median der $\mathrm{F}_{0}, 5 \%$ - und $95 \%$ Quantil der $\mathrm{F}_{0}$, Dauer des Sprechanteils) sowie Transkripte wurden mithilfe des PraatSkripts extrahiert (Schoenherr et al. 2021). Die Silbenzahl der Sprechanteile wurde mithilfe des R-packages sylly.de (Version 0.1-6) aus den Transkripten bestimmt. Die paraverbalen Parameter wurden für jeden Sprechanteil bestimmt, sodass für jede/jeden Patient_in/Probanden mehrere Messungen der Parameter vorlagen.

\section{Datenanalyse}

Die Auswertung der Daten erfolgte mit IBM SPSS Statistics 24 und R 4.1.1. Für die Analysen wurden nur vollständige Datensätze verwendet; fehlende Werte wurden mithilfe der "listwise deletion" ausgeschlossen. Für die Berechnung von $F_{0}$, Range $F_{0}$ und Sprechgeschwindigkeit mussten 5,46\% der Fälle aufgrund fehlender Stimmparameter ausgeschlossen werden. Für die Berechnung der Pausenlänge fehlten Daten von 3,8\% der Fälle. Für Gruppenvergleiche der deskriptiven Daten wurde je nach Verteilung der Daten parametrisch oder nichtparametrisch analysiert. Bei der Anwendung nichtparametrischer Daten wird statt des arithmetischen Mittels der Median der Skala berichtet. Zusätzlich dazu werden der Minimal- sowie Maximalwert angegeben, um die Verteilung besser zu charakterisieren. Fehlende Daten entstanden immer dann, wenn im Audiosignal zu viele überlagernde und störende Hintergrundgeräusche auftauchten; diese machten eine Differenzierung der Frequenzbänder unmöglich. Dementsprechend wurden auch diese ausgeschlossen. Aufgrund multipler Messungen der paraverbalen Merkmale pro Versuchsperson wurden die Zusammenhänge zwischen der Gruppenzugehörigkeit sowie den Fragebogenskalen (Depressivität, Ängstlichkeit, körperliche und psychische Gesundheit) und den paraverbalen Merkmalen mithilfe einzelner hierarchischer Regressionen (paraverbale Merkmale als Kriterien) analysiert. Zusätzlich wurde stets ein "random intercept" modelliert, um die intrain- dividuelle Variabilität der paraverbalen Merkmale verschiedener Sprechanteile zu beachten (Level 1: Messungen pro Patient_in; Level 2: Patient_innen). In allen Regressionen wurde der dichotome Prädiktor Geschlecht (0: männlich, 1: weiblich) eingeschlossen, um für geschlechtsspezifische Unterschiede zu kontrollieren (Biemans (2000), durchschnittliche $F_{0}$ der Männer bei ca. $125 \mathrm{~Hz}$; durchschnittliche $\mathrm{F}_{0}$ der Frauen bei ca. $200 \mathrm{~Hz}$ ).

\section{Ergebnisse}

Verglichen mit den Gesunden zeigten sich für die Patientengruppe mit Depression erwartungsgemäß höhere Werte für Depressivität $\left(M_{\text {Pat }}=15,47, S_{\text {Pat }} \pm 4,52 ; M_{K G}=3,40\right.$, $\left.\mathrm{SD}_{\mathrm{KG}} \pm 2,44 ; t(28)=-9,10, p<0,001\right)$ und Ängstlichkeit $\left(M_{\text {Pat }}=11,87, \mathrm{SD}_{\text {Pat }} \pm 3,29\right.$; $\mathrm{M}_{\mathrm{KG}}=1,73, \mathrm{SD}_{\mathrm{KG}} \pm 1,71 ; t(21,04)=-10,58$, $p<0,001)$. Außerdem hatten die $\mathrm{Pa}$ tient_innen geringere Ausprägungen der körperlichen ( $M_{\text {Pat }}=54,91, S_{\text {Pat }} \pm 8,33$; $M_{K G}=62,16, S_{K G} \pm 3,33 ; t(18,37)=3,13$, $p=0,004)$ und psychischen Gesundheit $\left(M_{\text {Pat }}=13,66, S_{\text {Pat }} \pm 5,29 ; M_{K G}=35,68\right.$, $\left.\mathrm{SD}_{\mathrm{KG}} \pm 2,87 ; t(28)=14,16, p<0,001\right)$. Die Sprechdauer der Patient_innen im ausgewählten Gesprächsausschnitt war nicht signifikant länger als der Sprechanteil der Kontrollprobanden (Dauer: Med $_{\text {Pat }}=85,55 \mathrm{~s}, \mathrm{SD}_{\text {Pat }} \pm 55,75$, Min Pat $=$ $18,42 \mathrm{~s}, \operatorname{MaX}_{\text {Pat }}=171,36 \mathrm{~s} ; \operatorname{Med}_{\mathrm{KG}}=55,43 \mathrm{~s}$, $\mathrm{SD}_{\mathrm{KG}} \pm 37,91, \operatorname{Min}_{\mathrm{KG}}=14,54 \mathrm{~s}, \mathrm{MaX}_{\mathrm{KG}}=$ $159,14 \mathrm{~s}, U(15,15)=80,00, p=0,19$; Silbenanzahl: Med $_{\text {Pat }}=321,00$, SD $_{\text {Pat }} \pm 303,60$, $\operatorname{Min}_{\text {Pat }}=99$, MaX Pat $_{\text {Pa }}=990 ;$ Med $_{K G}=301,00$, $\mathrm{SD}_{\mathrm{KG}} \pm 217,96, \mathrm{Min}_{\mathrm{KG}}=84, \operatorname{Max}_{\mathrm{KG}}=895$, $U(15,15)=93,00, p=0,44)$. Die Verteilungen der nichtparametrischen Skalen sind im Zusatzmaterial online: Abbildung S1 zu sehen. Die Häufigkeit einsilbiger Antworten (vorwiegend "Nein") variierte zwischen den Gruppen $\left(M_{\text {Pat }}=11,36\right.$, $\mathrm{SD}_{\text {Pat }} \pm 4,97, \mathrm{M}_{\mathrm{KG}}=7,43, \mathrm{SD}_{\mathrm{KG}} \pm 3,65$; $t(23,87)=-2,38, p=0,03)$.

Die Ergebnisse der Multi-Level-Analysen sind in - Tab. 1 zusammengefasst. Es zeigte sich ein signifikanter Gruppenunterschied in Bezug auf die Pausenlänge. Patient_innen machten signifikant längere Pausen (standardisierte Regressionskoeffizient $[\beta]=0,11, p=0,02, r=0,16)$. In Bezug auf die Fragebogenmaße fanden sich Zusammenhänge zwischen der Pau- senlänge und der psychischen Gesundheit $(\beta=-0,13, p<0,01, r=-0,18)$ sowie auch im Besonderen zur Ängstlichkeit $(\beta=0,12$, $p<0,01, r=0,17)$. Kürzere Pausen scheinen demnach mit psychischer Gesundheit zusammenzuhängen. Die Sprechgeschwindigkeit konnte signifikant negativ durch Depressivität $(\beta=-0,18, p=0,04$, $r=-0,23)$ und Ängstlichkeit $(\beta=-0,20$, $p<0,01, r=-0,25)$ vorhergesagt werden. Es konnten keine signifikanten Zusammenhänge in Bezug auf die $F_{0}$, die Spannweite der $F_{0}$ und die körperliche Gesundheit gefunden werden. Die einsilbigen Antworten hatten eine geringere Spannweite der $F_{0}$ (Mittelwert einsilbige Antworten: $30,25 \mathrm{~Hz}$, Mittelwert sonstige Antworten: $45,68 \mathrm{~Hz}, t(422,63)=6,00, p<0,01)$. Die „Intraclass-correlation"(ICC)-Werte zeigen, dass innerhalb der frequenzbasierten MaBe $\left(F_{0}\right.$, Spannweite der $\left.F_{0}\right)$ im Vergleich zur Pausenlänge eine höhere intraindividuelle Variabilität zu sehen ist. Diese scheint in den verschiedenen Sprechanteilen ein eher konstantes Merkmal innerhalb einer Person zu sein. Frauen weisen erwartungskonform generell höhere Grundfrequenzen und eine höhere Spannweiten der Grundfrequenz auf. In Bezug auf die Sprechgeschwindigkeit und Pausenlänge gibt es keine Geschlechtsunterschiede.

\section{Diskussion}

\section{Interpretation der Ergebnisse}

Die vorgestellten Ergebnisse zeigen, dass die Sprechgeschwindigkeit und teils die Pausenlänge auch in standardisierten Erhebungen mit Spontansprache Indikatoren für Ängstlichkeit und Depressivität sein können, da hier die wesentlichen Unterschiede zwischen depressiven Patient_innen und Gesunden deutlich wurden. Der Zusammenhang zwischen der Pausenlänge und Depressivität war nicht signifikant, weist aber explorativ auf eine mögliche Assoziation hin $(\beta=0,09$, $p=0,07, r=0,14)$. Im Hinblick auf die Depressivität zeigte sich, dass Patient_innen eine geringere Sprechgeschwindigkeit hatten. Auch die subjektive Ängstlichkeit konnte mit diesen Parametern in Verbindung gebracht werden. Eine stärkere Depressionsausprägung scheint mit einer monotoneren Sprechweise einher- 
zugehen. Dieser Zusammenhang zeigte sich jedoch ausschließlich explorativ zum einen im Gruppenunterschied bezüglich der Spannweite der $F_{0}(\beta=-0,12$, $p=0,08, r=-0,17)$ und zum anderen in der Regressionsanalyse der kontinuierlichen Depressivitätsvariable $(\beta=-0,12$, $p=0,09, r=-0,17$ ).

Bei Betrachtung der Depressionsausprägung wurde ersichtlich, dass die Verteilung in der klinischen Stichprobe sehr heterogen war. Möglicherweise waren einige der Patient_innen in ihrer Behandlung fortgeschritten, da die Patientengruppe in der Primärstudie lediglich danach ausgewählt wurde, ob zum Untersuchungszeitpunkt eine depressive Episode bestand. Hinzu kommt, dass die Fragebogenergebnisse bei 3 gesunden Probanden (20\% der Vergleichsgruppe) auf eine milde depressive Symptomatik hinwiesen. Die Überschneidung in der Verteilung von depressiven Patient_innen und gesunden Probanden könnte trotz der signifikanten Gruppenunterschiede in der Depressivität die geringen Effekte hinsichtlich der Gruppenunterschiede verursacht haben.

Das klinische Bild der Depression ist sehr heterogen, dementsprechend könnte es sinnvoll sein, paraverbale Merkmale in Bezug auf verschiedene Merkmale der Depression zu untersuchen. Dabei könnten die Zahl der depressiven Episoden, die Prominenz der Kardinalssymptome (Patient_innen mit vorwiegend trauriger Stimmung vs. Patient_innen mit Antriebslosigkeit und Gefühlsleere), die Schwere der Erkrankung sowie die medikamentöse Einstellung wichtige Einflussgrößen sein. Außerdem zeigen neuere Studien, dass nicht alle nonverbalen Merkmale (z.B. auch nicht alle Bewegungsparameter) für die Diagnostik der Depression gleich geeignet sind (Altmann et al. 2020b). Studienübergreifend besteht ein Zusammenhang zwischen Traurigkeit und einer geringen $F_{0}$ sowie einer geringen Spannweite der $F_{0}$ (Paeschke und Sendlmeier 2000). Da dieser Zusammenhang in Bezug auf die Depressivität nicht gezeigt werden konnte, kann angenommen werden, dass bei der vorliegenden Stichprobe nicht die niedergeschlagene Stimmung, sondern die Antriebslosigkeit im Vordergrund stand. Konform damit wurden signifikante Effekte in Bezug auf die Sprechgeschwindigkeit und explorative Befunde nachgewiesen; diese deuten auf einen Einfluss der Pausenlänge hin.

Bezogen auf die Spannweite der $\mathrm{F}_{0}$ könnte die vermehrte Beantwortung der Symptom- und Erkrankungsfragen im Interview mit "Nein", die empirisch zu einer Einschränkung der natürlichen Spannweite der $F_{0}$ geführt hat, ursächlich für die rein explorativen Befunde sein. Jedoch scheint gerade die Spannweite der $F_{0}$ ein wichtiger Indikator für Depressivität zu sein, da dieser Zusammenhang auch in bisheriger Forschung mehrheitlich gezeigt wurde (Cannizzaro et al. 2004; Mundt et al. 2007).

Höhere Depressivität in der Gesamtstichprobe war signifikant mit einer geringeren Sprechgeschwindigkeit assoziiert $(r=-0,23)$. Der Effekt ist als ein kleiner Effekt zu interpretieren. Mundt et al. (2007) haben einen stärkeren Zusammenhang zwischen Depressionsstärke und Sprechgeschwindigkeit gefunden $(r=-0,53)$. Verschiedene Untersuchungsabläufe und -charakteristika können die voneinander abweichenden Effektstärken erklären (Mundt et al. 2007): Erhebung über 4 Wochen mit Sicherung einer Baseline für die $\mathrm{F}_{0}(n=105)$. Die vorgestellten Ergebnisse lassen vermuten, dass die Pausen zwischen den Wörtern der Grund für die langsamere Sprechgeschwindigkeit sein können, was in vorliegender Forschung bereits beschrieben ist (Cannizzaro et al. 2004). Für zukünftige Forschung lässt sich ableiten, dass die Charakteristika, die zu einer veränderten Sprechgeschwindigkeit führen (e.g. Pausenlänge, Dauer des Sprechens der Vokale) wichtige Variablen sein können. Es ist nicht auszuschließen, dass sich ähnliche paraverbale Charakteristika auch bei anderen Störungen finden lassen. Ähnliche Befunde wurden für die untersuchte Ängstlichkeit erhoben. Da die Korrelation zwischen Depressivität und Ängstlichkeit in unserer Stichprobe sehr hoch ist, war es aufgrund des geringen Stichprobenumfangs nicht möglich, die Effekte differenzierter zu betrachten (e.g. durch die gleichzeitige Aufnahme der Parameter in das Regressionsmodell). Dies wäre ein Ansatzpunkt für zukünftige Forschung. Unter Hinzunahme einer größeren Stichprobe sowie mehrerer Indikatoren für psychische Gesundheit (e.g.
Depressivität, Ängstlichkeit, Misstrauen gegenüber Menschen etc.) könnten Zusammenhänge zu den paraverbalen Merkmalen gegeneinander in umfassenden Regressionsmodellen geprüft werden. Aktuell scheinen die Ergebnisse bezüglich Sprechgeschwindigkeit und Pausenlänge darauf hinzudeuten, dass diese beiden Parameter gute Indikatoren für eine generelle psychische Gesundheit sind. Da die bisherigen Untersuchungen zeigten, dass die Zusammenhänge von Depressivität und der Sprechgeschwindigkeit über verschiedene Wörter hinweg bestehen (Cummins et al. 2015), wird nicht davon ausgegangen, dass unterschiedliche Wortkonnotationen der Grund der gefundenen Unterschiede sind.

Da in der vorliegenden Arbeit nur der sprachliche Beitrag des Probanden betrachtet wurde, kann nicht ausgeschlossen werden, dass das Interaktionsmuster des Diagnostikers die Ergebnisse möglicherweise beeinflusst hat. Die aktuelle Forschung zeigt, dass Synchronie von paraverbalen Merkmalen im Therapieverlauf entsteht und es einen wechselseitigen Einfluss gibt. Die Angleichung von paraverbalen Merkmalen im psychotherapeutischen Gespräch bei sozialen Ängsten scheint interessanterweise eher einen negativen Einfluss auf den Behandlungserfolg zu haben (Schoenherr et al. 2021). Weitere Untersuchungsmöglichkeiten sind die Inaugenscheinnahme der Wirkung der paraverbalen Synchronie zwischen Proband und Interviewer oder die subjektive Wahrnehmung paraverbaler Unterschiede.

\section{Stärken und Limitationen der Studie}

Stärken der Studie sind die standardisierte Gesprächsgrundlage und trotzdem mögliche Antwort in Spontansprache, die eine Generalisierung auf natürliche Kontexte ermöglicht. Dabei ist anzumerken, dass die analysierten Sprechanteile nicht standardisiert wurden, was die Interpretation der Befunde aktuell erschwert. Weiterhin wurde die Analyse der paraverbalen Merkmale softwarebasiert durchgeführt und bei der weiteren Untersuchung die Multi-Level-Struktur der Daten beachtet.

Die Untersuchung weist aufgrund des kleinen Stichprobenumfangs Limitationen 
bezüglich der Generalisierbarkeit der Ergebnisse und bezüglich der Power auf. Da es sich um eine Sekundäranalyse handelt, konnte die Wirkung wichtiger Einflussfaktoren, die im Rahmen der Pilotstudie nicht erhoben wurden, wie etwa nichtklassifizierter Komorbiditäten oder Dauer der bereits erfolgten Therapie, nicht ausgeschlossen werden. Weiterhin hätte die Qualität der Aufnahmen durch Ansteckmikrophone und einen Leitfaden ohne geschlossene Fragen verbessert werden können. Offene Fragen hätten in der Gesprächssituation ein vermehrtes einsilbiges Antworten wie „Nein" vermieden.

\section{Fazit für die Praxis}

- Die Analyse paraverbaler Parameter im wissenschaftlichen Kontext ist attraktiv, da sie kostengünstig, nichtinvasiv und objektiv durchgeführt werden kann. Gerade paraverbale Merkmale, die die Flüssigkeit des Gesprächsverlaufs abbilden (z.B. Sprechgeschwindigkeit oder die Pausenlänge) mögen eine dienliche Ergänzung zu den verbalen Äußerungen des Patient_innen bieten.

- Werden diese Merkmale explizit definiert und in Diagnosemanuale aufgenommen, können Praktiker_innen schneller auch aufgrund paraverbaler Merkmale eine Diagnose stellen.

- Paraverbale Merkmale könnten die Informationssammlung ergänzen und trennschärfere Diagnosen ermöglichen.

- Treten eine geringe Sprechgeschwindigkeit und hohe Pausenlänge im Gespräch auf, sollte der/die Praktiker_in die Diagnosekriterien der Depression differenziert prüfen.

- Um paraverbale Merkmale im klinischen Kontext noch besser zu verstehen, sind weitere Studien notwendig.

\section{Korrespondenzadresse}

\section{Prof. Dr. Bernhard Strauß}

Institut für Psychosoziale Medizin, Psychotherapie und Psychoonkologie, Universitätsklinikum Jena

Stoystr. 3, 07743 Jena, Deutschland Bernhard.Strauss@med.uni-jena.de

Danksagung. Wir bedanken uns bei Prof. Dr. KarlJürgen Bär und Dr. Uta Pietsch, Psychiatrische Klinik des Universitätsklinikums Jena, für die Möglichkeit, Patient_innen in ihrer Klinik zu rekrutieren.

Förderung. Die Studie wurde im Kontext einer von der DFG (GZ: STR 306/28-3) geförderten Studie zu

\section{Sound of depression. Relationship between depressiveness and paraverbal features during the intake interview}

Background: Depression is characterized by specific vocal features in addition to cognitive, emotional, social and psychomotor impairments. To date, only a few studies have examined these in medical contexts and in contrast to healthy individuals. Research question: The relationship between depressiveness and paraverbal features was investigated in depressive patients and healthy subjects.

Methods: In a multistep procedure the audio content of anamnesis interviews with depressive $(n=15)$ and healthy subjects $(n=15)$ was annotated and transcribed using a software. The paraverbal features fundamental voice frequency, range of the voice, speech tempo and pause length were determined automatically. Hierarchical linear models were used to examine the influence of group membership, depressiveness, anxiety as well as mental and physical health on the paraverbal features.

Results: A correlation was found between depressiveness and speech rate. In addition, tendential correlations were found between the range of the fundamental frequency as well as the pause length and depressiveness. According to this, depressive patients are characterized by monotonous speech, a low speech tempo as well as longer pauses compared to healthy persons. Speech tempo and pause length were also associated with anxiety.

Discussion: Speech tempo, pause length, and span of the fundamental frequency appear to be relevant indicators for depressiveness and possibly anxiety. The span of the fundamental frequency is more depression-specific, whereas pause length and speech tempo are associated with depressiveness and anxiety. Future studies should examine the associations in larger samples of different clinical disorders.

\section{Keywords}

Speech production measurement · Voice analysis - Verbal behavior $\cdot$ Nonverbal communication . Anxiety

Untersuchung der Synchronie paraverbaler Merkmale zur Vorhersage des Therapieerfolgs durchgeführt.

Funding. Open Access funding enabled and organized by Projekt DEAL.

\section{Einhaltung ethischer Richtlinien}

Interessenkonflikt. A. Shugaley, U. Altmann, M. Brümmel, J. Meier, B. Strauß und D. Schönherr geben an, dass kein Interessenkonflikt besteht.

Alle beschriebenen Untersuchungen am Menschen oder an menschlichem Gewebe wurden mit Zustimmung der zuständigen Ethikkommission, im Einklang mit nationalem Recht sowie gemäß der Deklaration von Helsinki von 1975 (in der aktuellen, überarbeiteten Fassung) durchgeführt. Von allen beteiligten Patienten liegt eine Einverständniserklärung vor.

Open Access. Dieser Artikel wird unter der Creative Commons Namensnennung 4.0 International Lizenz veröffentlicht, welche die Nutzung, Vervielfältigung, Bearbeitung, Verbreitung und Wiedergabe in jeglichem Medium und Format erlaubt, sofern Sie den/die ursprünglichen Autor(en) und die Quelle ordnungsgemäß nennen, einen Link zur Creative Commons Lizenz beifügen und angeben, ob Änderungen vorgenommen wurden.

Die in diesem Artikel enthaltenen Bilder und sonstiges Drittmaterial unterliegen ebenfalls der genannten Creative Commons Lizenz, sofern sich aus der Abbil- dungslegende nichts anderes ergibt. Sofern das betreffende Material nicht unter der genannten Creative Commons Lizenz steht und die betreffende Handlung nicht nach gesetzlichen Vorschriften erlaubt ist, ist für die oben aufgeführten Weiterverwendungen des Materials die Einwilligung des jeweiligen Rechteinhabers einzuholen.

Weitere Details zur Lizenz entnehmen Sie bitte der Lizenzinformation auf http://creativecommons.org/ licenses/by/4.0/deed.de.

\section{Literatur}

Altmann U, Brümmel M, Meier J, Strauß B (2020a) Movement synchrony and facial synchrony as diagnosticfeatures of depression-A pilot study. JNerv Ment Dis 209(2):128-136. https://doi.org/ 10.1097/NMD.0000000000001268

Altmann U, Knitter LA, Meier J, Brümmel M, Strauß B (2020b) Nonverbale Korrelate depressiver Störungen: Eine Pilotstudie. Z Klin Psychol Psychother 49(4):1-10. https://doi.org/10.1026/ 1616-3443/a000602

Argyle M (1979) Körpersprache und Kommunikation. Junfermann, Paderborn

Balsters MJH, Krahmer EJ, Swerts MGJ, Vingerhoets AJJM (2012) Verbal and nonverbal correlates for depression: A review. Curr Psychiatry Rev 8(3):227-234. https://doi.org/10.2174/ 157340012800792966 
Biemans M (2000) Gender variation in voice quality (Netherlands Graduate School of Linguistics)

Boersma P (2001) Praat, a system for doing phonetics by computer. Glot Int 5(9):341-345

Bullinger M, Kirchberger I, Ware J (1995) Der deutsche SF-36 Health Survey Übersetzung und psychometrische Testung eines krankheitsübergreifenden Instruments zur Erfassung der gesundheitsbezogenen Lebensqualität. Z Gesundheitswiss 3(1):21-36. https://doi.org/10. 1007/BF02959944

Cannizzaro M, Harel B, Reilly N, Chappell P, Snyder PJ (2004) Voice acoustical measurement of the severity of major depression. Brain Cogn 56(1):30-35. https://doi.org/10.1016/j.bandc. 2004.05.003

Cummins N, Scherer S, Krajewski J, Schnieder S, Epps J, Quatieri TF (2015) A review of depression and suicide risk assessment using speech analysis. Speech Commun 71:10-49. https://doi.org/10. 1016/j.specom.2015.03.004

Faller H (2010) Kommunikation und Interaktion. In Faller H, Lang H (Hrsg) Medizinische Psychologie und Soziologie. Springer, Heidelberg, Berlin, New York, S202-211

Häuser W, Schmutzer G, Hinz A, Hilbert A, Brähler E (2013) Prävalenz chronischer Schmerzen in Deutschland. Schmerz 27(1):46-55. https://doi. org/10.1007/s00482-012-1280-z

Jablensky A, Kendell RE (2002) Criteria for assessing a classification in psychiatry. Psychiatric diagnosis and classification, Bd. 1

Juslin PN, Scherer KR (2005) Vocal expression of affect. Oxford University Press, Oxford

Kroenke K, Spitzer RL, Williams JB (2001) The PHQ-9: validity of a brief depression severity measure. J Gen Intern Med 16(9):606-613. https://doi.org/ 10.1046/j.1525-1497.2001.016009606.x

Lindemann K (2015) Emotionen in medizinischer Kommunikation. In: Busch A, Spranz-Fogasy T (Hrsg) Handbuch Sprache in der Medizin. Handbücher Sprachwissen, Bd. 11. De Gruyter, Berlin, S154-169

Mundt JC, Snyder PJ, Cannizzaro MS, Chappie K, Geralts DS (2007) Voice acoustic measures of depression severity and treatment response collected via interactive voice response (IVR) technology. J Neurolinguistics 20(1):50-64. https://doi.org/10.1016/j.jneuroling.2006.04. 001

Mundt JC, Vogel AP, Feltner DE, Lenderking WR (2012) Vocal acoustic biomarkers of depression severity and treatment response. Biol Psychiatry 72(7):580-587. https://doi.org/10.1016/j. biopsych.2012.03.015

Paeschke A, Sendlmeier WF (2000) Prosodic characteristics of emotional speech: Measurements of fundamental frequency movements. ISCA Tutorial and Research Workshop (ITRW) on speech and emotion

Pennacchini M, Pensieri C (2011) Is non-directive communication in genetic counseling possible? Clin Ter 162(5):141-144

Roter DL, Frankel RM, Hall JA, Sluyter D (2006) The expression of emotion through nonverbal behavior in medical visits. J Gen Intern Med 21(1):28-34. https://doi.org/10.1111/j.15251497.2006.00306.x

Schoenherr D, Strauss B, Stangier U, Altmann U (2021) The influence of vocal synchrony on outcome and attachment anxiety/avoidance in treatments of social anxiety disorder. Psychotherapy. https:// doi.org/10.1037/pst0000393
Schuller B, Steidl S, Batliner A, Burkhardt F, Devillers L, Müller C, Narayanan S (2013) Paralinguistics in speech and language: State-of-the-art and the challenge. Comput Speech Lang 27(1):4-39. https://doi.org/10.1016/j.csl.2012.02.005

Spitzer RL, Kroenke K, Williams JB, Löwe B (2006) $A$ brief measure for assessing generalized anxiety disorder: The GAD-7. Arch Intern Med 166(10):1092-1097. https://doi.org/10.1001/ archinte.166.10.1092

Stassen HH (1995) Affekt und Sprache: Stimm-und Sprachanalysen bei Gesunden, depressiven und schizophrenen Patienten Bd. 79. Springer, Heidelberg, Berlin, New York https://doi.org/10 1007/978-3-642-79726-2

Taguchi T, Tachikawa H, Nemoto K, Suzuki M, Nagano T, Tachibana R, Nishimura M, Arai T (2018) Major depressive disorder discrimination using vocal acoustic features. J Affect Disord 225:214-220 https://doi.org/10.1016/j.jad.2017.08.038

Tonti M, Gelo OC (2016) Rate of speech and emotionalcognitive regulation in the psychotherapeutic process: a pilot study. Res Psychother. https:// doi.org/10.4081/ripppo.2016.232

Weeks JW, Lee C-Y, Reilly AR, Howell AN, France C, Kowalsky JM, Bush A (2012) "The Sound of Fear": Assessing vocal fundamental frequency as a physiological indicator of social anxiety disorder. J Anxiety Disord 26(8):811-822

Westland G (2015) Verbal and non-verbal communication in psychotherapy. WW Norton, New York

Wiltshire TJ, Philipsen JS, Trasmundi SB, Jensen TW, Steffensen SV (2020) Interpersonal coordination dynamics in psychotherapy: a systematic review. Cognit Ther Res 44:752-773. https://doi.org/10. 1007/s10608-020-10106-3
Hier steht eine Anzeige. Springer 